\title{
Excitation and Initiation of Impulse
}

Part III. Accommodation

By

\section{Kazumi Yamagiwa}

(山極 一三)

From the Department of Physiology, Tokyo Medical and Dental University, Yushima, Bunkyo-ku, Tokyo

(Received for publication, October 28, 1956)

As stated in Parts I and II, distinction of excitation from initiation of impulse is very useful for understanding several phenomena of excitation, particularly some phenomena of "accommodation", without introducing the very concept. This does never mean, however, that accommodation does not occur. As a matter of fact, some rise of $I_{r}$ has been the case near ${ }^{1-3)} \beta_{x}$. The point is whether the observed rise of $I_{r}$ was due to rise of $i_{r}$ or of $m$, because $\mathrm{I}_{\mathrm{r}}=m \eta i_{r}$ and $\eta=1+\frac{m-1}{m} \frac{1}{\mathrm{e}^{\frac{\mathrm{D}}{\alpha}}-1}$ (eq. (5), Part I). We will examine the matter in various cases, below.

I. Normal $\beta_{\mathrm{r}}$ and $\frac{\mathrm{I}_{\infty}}{\mathrm{I}_{\mathrm{r}}}-\gamma$-line

1. In relation to $i_{r}$-rise, supposed

1) On $\beta_{\mathbf{r}}$. If $i_{r}$ really rises near $\beta_{\mathbf{r}}$, then it will be reasonable to suppose that it will do so in larger $\beta$, too, even if little in magnitude. Let us consider therefore $\mathrm{i}_{\mathrm{r}}(\beta)$, the rheobase as a function of $\beta$, and put

$$
\begin{aligned}
& i_{\mathrm{r}}(\beta)=i_{r} f(\beta), \\
& i_{r}: \text { the value of } i_{\mathbf{r}}(\beta) \text { for } \beta=\infty \text { (rectangular pulse), } \\
& f(\beta): \text { a function of } \beta \text {, which is practically unity in large } \beta \text {, then grows gradual- } \\
& \text { ly with decrease of } \beta .
\end{aligned}
$$

Now, the gradient is $\beta$ at $\mathrm{x}=0$ and $\frac{\beta}{m}$ at $\mathrm{x}=\mathrm{L}$, hence $\mathrm{i}_{\mathrm{r}}(\beta)$ should be $i_{r} f(\beta)$ and $i_{r} f\left(\frac{\beta}{m}\right)$ respectively. However, $m$ is very near unity in normal conditions, so we may disregard this difference and consider just the difference due to variation of $\beta$ itself.

Assuming $m, \mathrm{D}$ and $\eta$ constant, and neglecting $\varepsilon$ (see Part I) as before, we have for the limiting case

$$
\Delta \mathrm{t}=\mathrm{t}_{2}-\mathrm{t}_{1}=\frac{m i_{\mathrm{r}} f\left(\beta_{\mathrm{r}}\right)}{\beta_{\mathrm{r}}}-\frac{i_{\mathrm{r}} f\left(\beta_{\mathrm{r}}\right)}{\beta_{\mathrm{r}}}=\frac{i_{\mathrm{r}} f\left(\beta_{\mathrm{r}}\right)}{\beta_{\mathrm{r}}}(m-1)=\mathrm{D}
$$


Hence, $\quad \beta_{\mathbf{r}}=\frac{i_{r} f\left(\beta_{\mathrm{r}}\right)(m-1)}{\mathrm{D}}=\frac{\mathrm{I}_{\mathrm{r}}}{\eta \mathrm{D}} \frac{m-1}{m} f\left(\beta_{\mathrm{r}}\right)=$ eq. (1) $($ Part II $) \times f\left(\beta_{\mathrm{r}}\right)$

In other words, $\beta_{\mathrm{r}}$ gets $f\left(\beta_{\mathrm{r}}\right)$-times larger than when the rhobase is invariable.

Now, eq. (1) has a meaning only when $f\left(\beta_{\mathrm{r}}\right)$ is finite. Actually, $\mathbf{I}_{\mathrm{r}}$ at $\beta_{\mathrm{r}}$ was found finite $(1.2-1.3$ times normal) as stated in Part II. This means that $f\left(\beta_{\mathrm{r}}\right)=1.2-1.3$, accordingly, that $\beta_{\mathrm{r}}$ expressed by eq. (1) can be about as large as the standard value (see eq. (2), Part II). However, the finiteness of $\mathrm{I}_{\mathrm{r}}$ at $\beta_{\mathrm{r}}$, as a fact as well as a theoretical claim, contradicts strongly to the classical idea, which predicts $\infty$. Another serious point that a slight reduction of $\beta$ makes the current suddenly ineffective, was described in Part II. A discontinuous change of the rheobase, from 1.2-1.3 normal to $\infty$, might not be impossible, but more probably there is something else still undiscovered there. This is nothing but the condition $\Delta \mathrm{t} \leqq \mathrm{D}$, if viewed from the present theory.

2) On $\frac{I_{\infty}}{I_{r}}-\gamma$-line. We take here the rheobase as a function of the rate of increase of the current. The rate is determined by $\mathrm{I}_{\infty}$ and $\gamma$, and also by time. For convenience, we will take the initial slope $\delta\left(=\frac{\mathrm{I} \infty}{\gamma}\right)$ and put $i_{\mathrm{r}}(\delta)=i_{r} \mathrm{~F}(\delta)$,

$$
\begin{aligned}
& i_{r} \text { : the value of } i_{\mathrm{r}}(\delta) \text { for } \delta==\infty \text { (rectangular pulse), } \\
& F(\delta)=F\left(\frac{\mathrm{I} \infty}{\gamma}\right): \text { a function similar to } f(\beta) .
\end{aligned}
$$

For the points $\mathrm{x}=0$ and $\mathrm{x}=\mathrm{L}$, we should put, strictly,

$$
\text { and } \quad \begin{aligned}
\mathrm{I}_{\infty}\left(1-\mathrm{e}^{-\frac{\mathrm{t}_{\mathbf{1}}}{r}}\right) & =i_{r} \mathrm{~F}(\delta), \\
\frac{\mathrm{I}_{\infty}}{m}\left(1-\mathrm{e}^{-\frac{\mathbf{t}_{2}}{r}}\right) & =i_{r} \mathrm{~F}\left(\frac{\delta}{m}\right) .
\end{aligned}
$$

But if taken $\mathrm{F}(\hat{\boldsymbol{o}}) \fallingdotseq \mathrm{F}\left(\frac{\boldsymbol{\delta}}{\mathrm{m}}\right)$ as in the case of linearly rising current, then

In the limiting case

$$
t_{1}=r \ln \frac{\mathrm{I}_{\infty}}{\mathrm{I}_{\infty}-i_{r} \mathrm{~F}(\delta)} \text { and } \mathrm{t}_{2}=r \ln \frac{\mathrm{I}_{\infty}}{\mathrm{I}_{\infty}-m i_{r} \mathrm{~F}(\boldsymbol{\delta})}
$$

The solution is

$$
\Delta \mathrm{t}=\mathrm{t}_{2}-\mathrm{t}_{1}=\gamma \ln \frac{\mathrm{I}_{\infty}-i_{r} \mathrm{~F}(\delta)}{\mathrm{I}_{\infty}-m i_{r} \mathrm{~F}(\delta)}=\mathrm{D}
$$

$$
\frac{\mathrm{I}_{\infty}}{i_{r}}\left(=\frac{\mathrm{I} \infty \eta m m}{\mathrm{I}_{\mathrm{r}}}\right)=\mathrm{F}(\boldsymbol{\delta}) \frac{m \mathrm{e}^{-\frac{\mathrm{D}}{r}}-1}{\mathrm{e}^{\frac{\mathrm{D}}{r}}-1}=\mathrm{F}(\boldsymbol{\delta})\left(m+\frac{m-1}{\mathrm{e}^{\frac{\mathrm{D}}{r}}-1}\right)
$$




$$
\begin{aligned}
& \fallingdotseq \mathrm{F}(\delta)\left\{m+(m-1) \frac{\gamma}{\mathrm{D}}\right\}\left(\frac{\mathrm{l}}{\left(\mathrm{e}^{\frac{\mathrm{D}}{\gamma}}-1\right.} \fallingdotseq \frac{\gamma}{\mathrm{D}} \text { introduced }\right) \\
& \therefore \quad \frac{\mathrm{I}_{\infty}}{\mathrm{I}_{\mathrm{r}}}=\frac{\mathrm{F}(\boldsymbol{\delta})}{\eta}\left(1+\frac{m-1}{m} \frac{\gamma}{\mathrm{D}}\right)
\end{aligned}
$$

By assumption, $F(\delta)$ is a function which, growing from unity, gets larger with decrease of $\delta$. We cannot foretell precisely how $\delta$ will change with $\gamma$, because we do not know how $\mathrm{I}_{\infty}$ will change with $\gamma$, this being the very thing which we wish to clarify. But it is certain that $\delta$ starts with a very large value, and, decreasing with increase of $\gamma$, reaches a certain value, $\delta_{\mathrm{r}}$, which corresponds to $\beta_{\mathrm{r}}$. $i_{\mathrm{r}}(\delta)$ will then be $i_{\boldsymbol{r}} \mathrm{F}\left(\delta_{\mathrm{r}}\right)$. If taken $\mathrm{F}\left(\boldsymbol{\delta}_{\mathrm{r}}\right)=f\left(\beta_{\mathrm{r}}\right)=1.2-1.3$, then eq. (2) may be said to be practically linear, although never so strictly except in the range of large $\delta$ where $F(\delta) \fallingdotseq 1$.

2. In relation to $m$-rise, supposed

A slowly rising cathodic current will probably loosen the membrane and thus diminish the resistance $r_{m}$ (see Suzuki $\left.{ }^{4}\right)$. This makes $\mathrm{k}$ smaller, which makes L larger in turn (see Fig. 1, Part I). A further enlargement of $\mathrm{L}$ is expected from reduction of $f$ due to diminution of $\mathrm{V}$ amounting to $50 \%$ of the normal at $\beta_{\mathrm{r}}{ }^{1}$. . There are also factors which act to diminish $\mathrm{L}$, namely, ${ }^{1)}$ enlargement of $f$ by decrease of $\mathrm{r}_{\mathrm{m}}{ }^{5)}$, and 2) some possible decrease of $k^{\prime}$, although presumably very little. Even if $\mathrm{L}$ remains unchanged after all these changes, still $m$ can augment as the result of $\mathrm{k}$-decrease alone. The rise of $m$ seems thus highly probable.

1) On $\beta_{\mathbf{r}}$. Assume $i_{r}, \mathrm{D}, \alpha$ and practically also $\eta$ constant, and further

$$
\mathrm{m}(\beta)=m f(\beta) \text {, }
$$

$m$ : the value of $m(\beta)$ for $\beta=\infty$ (rectangular pulse),

$f(\beta)$ : the same function as before.

From $\mathrm{I}_{0}=\beta \mathrm{t}_{1}=i_{r}$ and $\mathrm{I}_{\mathrm{L}}=\frac{\beta}{m f(\beta)} \mathrm{t}_{2}=i_{r}$, and under neglect of $\varepsilon$ as before, we have for the limiting case

$$
\begin{gathered}
\Delta \mathrm{t}=\mathrm{t}_{\mathbf{2}}-\mathrm{t}_{\mathbf{1}}=\frac{i_{\mathbf{r}}}{\beta_{\mathrm{r}}}\left\{m f\left(\beta_{\mathrm{r}}\right)-1\right\}=\mathrm{D} ; \\
\beta_{\mathrm{r}}=\frac{i_{\mathbf{r}}}{\mathrm{D}}\left\{m f\left(\beta_{\mathrm{r}}\right)-1\right\}=\frac{\mathrm{I}_{\mathrm{r}}}{\eta \mathrm{D}} \frac{m f\left(\beta_{\mathrm{r}}\right)-1}{m} \\
\text { or }=\frac{\mathrm{I}_{\mathrm{r}}}{\eta \mathrm{D}} \frac{m-1}{m} \frac{m f\left(\beta_{\mathrm{r}}\right)-1}{m-1}=\mathrm{eq} . \text { (1) (Part II) } \times \frac{m f\left(\beta_{\mathrm{r}}\right)-1}{m-1}
\end{gathered}
$$

In a standard case, eq. (1) (Part II) turns into eq. (2) (Part II), which gives $\beta_{\mathrm{r} . \mathrm{o}}=0.6 \mathrm{mV} / \mathrm{msec}$. Hence, if taken $f\left(\beta_{\mathrm{r}}\right)=1.2-1.3$ in eq. (3) above, then $\beta_{\mathrm{r}} \fallingdotseq 0.6 \times(11-16) \fallingdotseq 7-10 \mathrm{mV} / \mathrm{msec}$, which is larger than the largest value obtained by Diecke ${ }^{1)}$. This suggests that rise of $m$ in linearly 
rising current is improbable, as long as $f\left(\beta_{\mathrm{r}}\right)$ is so large as 1.2-1.3. stant.

2) On $\frac{\mathrm{I}_{\infty}}{\mathrm{I}_{\mathrm{r}}}-\gamma$-line. Suppose that $\mathrm{m}(\boldsymbol{\delta})=m \mathrm{~F}(\boldsymbol{\delta})$, and $i_{r}, \mathrm{D}$ and $\eta$ conFrom $\mathrm{I}_{\infty}\left(1-\mathrm{e}^{-\frac{\mathrm{t}_{1}}{r}}\right)=i_{r}$ and $\frac{\mathrm{I}_{\infty}}{m \mathrm{~F}(\delta)}\left(1-\mathrm{e}^{-\frac{\mathrm{t}_{2}}{r}}\right)=i_{r}$, we have

$$
\begin{aligned}
& \mathrm{t}_{1}=r \ln \frac{\mathrm{I}_{\infty}}{\mathrm{I}_{\infty}-i_{r}} \text { and } \mathrm{t}_{2}=r \ln \frac{\mathrm{I}_{\infty}}{\mathrm{I}_{\infty}-i_{r} m \mathrm{~F}(\delta)} ; \\
& \Delta \mathrm{t}=\gamma \ln \frac{\mathrm{I}_{\infty}-i_{r}}{\mathrm{I}_{\infty}-i_{r} m \mathrm{~F}(\delta)}=\mathrm{D} ; \\
& \frac{\mathrm{I}_{\infty}}{i_{r}}=\frac{m \mathrm{~F}(\delta) \mathrm{e}^{\frac{\mathrm{D}}{r}}-1}{\mathrm{e}^{\frac{\mathrm{D}}{r}}-1}=m \mathrm{~F}(\delta)+\frac{m \mathrm{~F}(\delta)-1}{\mathrm{e}^{\frac{\mathrm{D}}{r}}-1}
\end{aligned}
$$

Introducing $\eta m i_{r}=\mathrm{I}_{\mathrm{r}}$ and $\frac{1}{\mathrm{e}^{\frac{\mathrm{D}}{\gamma}}-1} \fallingdotseq \frac{\gamma}{\mathrm{D}}$

$$
\frac{\mathrm{I}_{\infty}}{\mathrm{I}_{\mathrm{r}}}=\frac{\mathrm{F}(\boldsymbol{\delta})}{\eta}\left(1+\frac{m-\frac{1}{\mathrm{~F}(\boldsymbol{\delta})}}{m} \frac{\gamma}{\mathrm{D}}\right)
$$

Eq. (4), like (2), can be linear in the range of $\mathrm{F}(\delta) \doteqdot 1$ only. The final slope is larger than that of eq. (2), so the linearity is the worse as a whole.

\section{Consideration}

Two important conclusions are drawn from the above examinations:

1) A rise of $\mathrm{I}_{\mathrm{r}}$, whether due to rise of $i_{r}$ or $m$, not only contradicts to the appearance of $\beta_{\mathrm{r}}$ of a reasonable value, but also disturbs the linearity

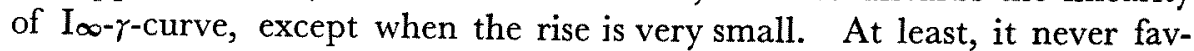
ours the appearance of these lines, as was foreseen already in Part I.

2) The rise of $I_{\mathrm{r}}$ near $\beta_{\mathrm{r}}$ appears to be due to rise of $i_{r}$, not of $m$. This is, however, because $f\left(\beta_{\mathrm{r}}\right)$ is so large as $1.2-1.3$. If it is smaller, then the matter turns out quite different. The possibility will be stated in Discussion below, but before that we will examine the matter in some other cases.

\section{II. $\mathrm{I}_{\mathrm{r}}$ and $\beta_{\mathrm{r}}$ in Electrotonus}

It is known that $\mathrm{I}_{\mathrm{r}}$ and $\beta_{\mathrm{r}}$ both rise in long-lasting C.E.T. (Tasaki $\left.{ }^{6}\right)$ ). A remarkable thing thereby is that the rise of $I_{r}$ is just little while that of $\beta_{\mathrm{r}}$ enormous. In Tasaki's observation above quoted, "the rheobase was not appreciably changed " but $\beta_{\mathrm{r}}$ changed from $\frac{1}{62}$ - to $\frac{1}{18}-\mathrm{msec}^{-1}$, that is, to about 3.5-times as large. 
If wanted to understand this unproportionality from Hill's theory, we have to consider two events, a small rise of the rheobase and a large reduction of $\lambda$, taking place simultaneously. But if viewed from the present theory, it is comprehensive as a simple result of $m$-rise only :

Eq. (5) (Part I) : $\mathrm{I}_{\mathrm{r}}=m_{\eta} i_{r}$

Eq. (1) (Part III) : $\beta_{\mathrm{r}}=\frac{i_{r}(m-1)}{\mathrm{D}} f\left(\beta_{\mathrm{r}}\right)\left(i_{r}\right.$ varying with $\left.\beta\right)$

Eq. (3) (Part III) $: \beta_{\mathbf{r}}=\frac{i_{\mathbf{r}}}{\mathrm{D}}\left\{m f\left(\beta_{\mathbf{r}}\right)-1\right\} \quad(m$ varying with $\beta)$.

If C.E.T. raises $i_{r}$, then both $I_{r}$ and $\beta_{r}$ should rise in the same proportion, but if it raises $m$, then $I_{\mathbf{r}}$ rises just proportionally as before, while $\beta_{\mathrm{r}}$ very much more. Quantitatively, eq. (1) (Part III) is satisfactory, because a rise of $m$ by just 5\% (from 1.02 to 1.07) suffices to explain Tasaki's result. Eq. (3) (Part III) is not available, again because $f\left(\beta_{\mathrm{r}}\right)$ is so large as $1.2-1.3$.

If the above consideration is correct, then in A.E.T. a little decrease of $m$ (from 1.02 to 1.00 at most), accordingly of $\mathrm{I}_{\mathrm{r}}$, and a relatively large diminution of $\beta_{\mathbf{r}}$ are expected. In actuality, $\beta_{\mathbf{r}}$ decreased to $\frac{1}{900} \mathrm{msec}^{-1}$, but $I_{r}$ increased from 25 to $100 \mathrm{mV}$ in Tasaki's experiment above quoted. The reason of this discrepancy is not clear, but one might be that in Tasaki the stimulus was expressed in voltage, which could have acted only through the membrane resistance, which must have been increased in A.E.T.

III. $I_{r}$ and $\beta_{\mathbf{r}}$ in Low Temperature

As quoted in Part II, $\mathrm{I}_{\mathrm{r}}$ changes very little but $\beta_{\mathrm{r}}$ gets very small in low temperature (Tasaki ${ }^{7)}$ ). We have here another example of unproportional change of $I_{r}$ and $\beta_{r}$. It must be due partly to prolongation of $D$ as stated before, but very probably also to diminution of $m$, which, if occurred in a standard case, cannot be large only from 1.02 to 1.0 at most), but still can bring forth an enormous decrease of $\beta_{\mathrm{r}}$.

\section{IV. $\mathrm{I}_{\mathrm{r}}$ and $\beta_{\mathrm{r}}$ in Relation to $\mathrm{Na}^{*}$-concentration}

It may not be appropriate to discuss the complicated effects of ions upon excitability, on the same line with that of electric current. Here just one example which is very interesting in connextion with the present theory, will be quoted.

According to Diecke ${ }^{1}$, both $\mathrm{I}_{\mathrm{r}}$ and $\beta_{\mathrm{r}}$ decrease with increase of $\mathrm{Na}$ concentration of the bathing fluid. Here again, the decrease of $\beta_{\mathrm{r}}$ is much more marked than that of $\mathbf{I}_{\mathbf{r}}$ (Abb. 11). For example, at $\left[\mathrm{Na}^{\cdot}\right]=444 \mathrm{mM} / \mathrm{l}$, $\beta_{\mathrm{r}}$ was already so small as $44 \%$, while $\mathrm{I}_{\mathrm{r}}$ remained at about $80 \%$, of the 
normal (see Tab. 1 and Abb. 11). This unproportional change of $I_{r}$ and $\beta_{\mathrm{r}}$ seems to point to $m$-change taking place in this case, too. In Diecke's preparations, the value of $m$ is seemingly near about 1.2 as stated in Part II. If $m$ ran down to 1.1 in the above example, $I_{r}$ must have decreased by about $10 \%$, while $\beta_{\mathrm{r}}$ by just $50 \%$ (eq. (1), Part II), which is about in accordance with the actual observation. Why $m$ decreases in higher $\mathrm{Na}$ concentrations, is not clear, but decrease of $\mathrm{L}$ due to increase of $\mathrm{V}$, accordingly of $f$, the safety factor, must be a powerful agent.

\section{Repetitive Responses \\ 1. To constant current}

The limiting condition for initiation of impulse is

$$
\text { Eq. (4) (Part I) : } \Delta \mathrm{t}=\alpha \ln \frac{\mathrm{I}_{0}-i_{r}}{\mathrm{I}_{0}-m i_{r}}=\mathrm{D}
$$

In Part $I$, the characteristics of the repetitive responses were interpreted as due to gradual rise of $\alpha$ with time. However, $\mathbf{I}_{0}$ being long-lasting, there are two other possibilities, namely, rise of $i_{r}$ and $m$. If they increase, they exert just similar effects as $\alpha$-increase upon $t_{2}$ and $\Delta t$, so that the known features of repetitive responses can be explained similarly. Here, just some consideration will be carried out on the time-length, during which the response is repeated.

1) Rise of $i_{r}$ with time and $\mathrm{I}_{0}$, supposed, under $m, \mathrm{D}$, a constant. Replacing $i_{r}$ with $i_{r}\left(I_{0}, t\right)$ in eq. (4) (Part $\left.I\right)$, we have

$$
\begin{aligned}
& \qquad I_{0}-i_{r}\left(I_{0}, t\right)=e^{\frac{D}{\alpha}}\left(I_{0}-m i_{r}\left(I_{0}, t\right)\right), \\
& \text { from which } \left.i_{r}\left(I_{0}, t\right)=I_{0} \frac{e^{\frac{D}{\alpha}}-1}{m e^{\frac{D}{\alpha}}-1}=I_{0} \frac{1}{y^{m}} \text { (see eq. (5), Part } I\right) \text { This is the rela- }
\end{aligned}
$$

tion between $i_{r}$ (raised) and $\mathrm{I}_{0}$ (applied) when the response is just stopping.

Put now $i_{\mathbf{r}}\left(\mathrm{I}_{0}, \mathrm{t}\right)=i_{r} f\left(\mathrm{I}_{0}, \mathrm{t}\right)$, where $i_{r}$ is the value of $\mathrm{i}_{\mathbf{r}}\left(\mathrm{I}_{0}, \mathrm{t}\right)$ at $\mathrm{t}=\mathrm{o}$ and $f\left(\mathrm{I}_{0}, \mathrm{t}\right)$ a function growing from unity, and introduce it into the above equation. Then

$$
f\left(\mathrm{I}_{0}, \mathrm{t}\right)=\frac{\mathrm{I}_{0}}{i_{r} \eta m}=\frac{\mathrm{I}_{0}}{\mathrm{I}_{\mathrm{r}}}
$$
changed

2) Rise of $m$ with time and $I_{0}$, supposed, under other variables un-

Replacing $m$ in eq. (4) (Part $\mathrm{I})$ with $\mathrm{m}\left(\mathrm{I}_{0}, \mathrm{t}\right)$, we have

$$
\begin{aligned}
& \mathrm{m}\left(\mathrm{I}_{0}, \mathrm{t}\right) i_{r} \mathrm{e}^{\frac{\mathrm{D}}{\alpha}}=\mathrm{I}_{0} \mathrm{e}^{\frac{\mathrm{D}}{\alpha}}-\mathrm{I}_{0}+i_{r} \\
& \mathrm{~m}\left(\mathrm{I}_{0}, \mathrm{t}\right)=\frac{\mathrm{I}_{0}}{i_{r}}\left(1-\mathrm{e}^{-\frac{\mathrm{D}}{\alpha}}\right)+\mathrm{e}^{-\frac{\mathrm{D}}{\alpha}}=\frac{\mathrm{I}_{0}}{\mathrm{I}_{\mathrm{r}}} m \eta\left(1-\mathrm{e}^{-\frac{\mathrm{D}}{\alpha}}\right)+\mathrm{e}^{-\frac{\mathrm{D}}{\alpha}}
\end{aligned}
$$


Putting $\mathrm{m}\left(\mathrm{I}_{0}, \mathrm{t}\right)=m f\left(\mathrm{I}_{0}, \mathrm{t}\right), m$ being the value of $\mathrm{m}\left(\mathrm{I}_{0}, \mathrm{t}\right)$ at $\mathrm{t}=0$,

$$
f\left(\mathrm{I}_{0}, \mathrm{t}\right)=\frac{\mathrm{I}_{0} \eta}{\mathrm{I}_{\mathrm{r}}}\left(1-\mathrm{e}^{-\frac{\mathrm{D}}{\alpha}}\right)+\frac{1}{m} \mathrm{e}^{\frac{\mathrm{D}}{\alpha}}
$$

The values of $t$ which satisfy eq. (5) or (6) respectively determine the "total adaptation time, T" in Granit and Skoglund's terminology". Presumably, eq. (6) will fit the observations better, because the case under examination is a special case of C.E.T., where the rise of $m$ seems certain, as stated before. Unfortunately, the form of $f\left(\mathrm{I}_{0}, \mathrm{t}\right)$ is unknown, so we cannot formulate $T$ as a function of $I_{0}$, but it is interesting to make a simple assumption that $f\left(\mathrm{I}_{0}, \mathrm{t}\right)=f(\mathrm{t})$, that is, that $i_{r}$ or $m$ is a function of $\mathrm{t}$ only, and to consider two special cases, 1) $f(\mathrm{t})=\mathrm{e}^{\frac{\mathrm{t}}{\mathrm{p}}}$, p constant, and 2) $f(\mathrm{t})=\ln (\mathrm{e}$ $\left.+\frac{\mathrm{t}}{\mathrm{q}}\right) \fallingdotseq \ln \frac{\mathrm{t}}{\mathrm{q}}$ in large $\frac{\mathrm{t}}{\mathrm{q}}$. The former, combined with eq. (5), leads to $\mathrm{t}=$ $\mathrm{p} \ln \frac{\mathrm{I}_{0}}{\mathrm{I}_{\mathrm{r}}}$. If $\mathrm{p}$ is replaced by $\lambda$, then we have just the equation derived by $\mathrm{Katz}^{9)}$ on the basis of Hill's theory. Katz's equation represents therefore a special case where $f(\mathrm{t})=\mathrm{e}^{\frac{\mathrm{t}}{\lambda}}$ is introduced, which, in view of the present theory, has no proper reason. It is not strange that it does not fit the observations well ${ }^{8)}$. On the other hand, the latter combined with eq. (6) leads to

$$
\begin{aligned}
& \ln \mathrm{t}=\frac{\mathrm{I}_{0}}{\mathrm{I}_{\mathrm{r}}} \eta\left(1-\mathrm{e}^{-\frac{\mathrm{D}}{\alpha}}\right)+\frac{1}{m} \mathrm{e}^{-\frac{\mathrm{D}}{\alpha}}+\ln \mathrm{q}=\mathrm{c}_{1} \frac{\mathrm{I}_{0}}{\mathrm{I}_{\mathrm{r}}}+\mathrm{c}_{2} \\
& \left(\mathrm{c}_{1} \Rightarrow \eta\left(1-\mathrm{e}^{-\frac{\mathrm{D}}{\alpha}}\right) ; c_{2}=\frac{1}{m} \mathrm{e}^{-\frac{\mathrm{D}}{\alpha}}+\ln \mathrm{q}\right),
\end{aligned}
$$

a linear relationship between $\ln t$ and $\frac{I_{0}}{I_{r}}$ as long as $\frac{D}{\alpha}$ remains unchanged. This supports Granit and Skoglund ${ }^{8)}$ who demonstrated a practical linearity of the $\ln \mathrm{T}-\frac{\mathrm{I}_{0}}{\mathrm{I}_{\mathrm{r}}}$-curve, and suggests, conversely, a logarithmic rise of $m$ with t strongly.

\section{To linearly rising current}

Eq. (3) (Part II) was derived from eq. (4) (Part I), regarding $i_{r}$ and $m$ constant. Here we assume them to be a function of $\beta$ and $\mathrm{T}$.

1) $\mathbf{i}_{\mathbf{r}}(\beta, \mathbf{T})=i_{\boldsymbol{y}} f(\beta) \mathbf{F}(\mathrm{T})$, supposed

$i_{r}$ : the value of $i_{r}(\beta, T)$ at $\beta=\infty$ and $T=0$,

$f(\beta)$ : the same function as before,

$F(T)$ : a function of $T$ growing from unity; the end of $t_{0}$ taken as origin of T (Fig. 2, Part II) 
Assume $m=$ constant, and consider the case of $\beta=\beta_{\mathbf{r}}$. Then, in the same way as in Part II,

$$
\begin{aligned}
& \Delta \mathrm{t}_{\mathrm{n}}=\alpha \ln \frac{i_{r}+\beta_{\mathrm{r}} \mathrm{T}_{\mathrm{n}-1}-i_{r} f\left(\beta_{\mathrm{r}}\right) \mathrm{F}\left(\mathrm{T}_{\mathrm{n}}\right)}{i_{r}+\beta_{r} \mathrm{Tn}_{-1}-i_{r} m f\left(\beta_{\mathrm{r}}\right) \mathrm{F}\left(\mathrm{T}_{\mathrm{n}}\right)} \\
& =a \ln \frac{1-\frac{i_{r} f\left(\beta_{\mathrm{r}}\right) \mathrm{F}\left(\mathrm{T}_{\mathrm{n}}\right)}{\beta_{\mathrm{r}} \mathrm{T}_{\mathrm{n}-1}}}{1-\frac{i_{r} m f\left(\beta_{\mathrm{r}}\right) \mathrm{F}\left(\mathrm{T}_{\mathrm{n}}\right)}{\beta_{\mathrm{r}} \mathrm{T}_{\mathrm{n}-1}}}\left(i_{r} \text { neglected against } i_{r} f\left(\beta_{\mathrm{r}}\right) \mathrm{F}\left(\mathrm{T}_{\mathrm{n}}\right)\right) \\
& =\alpha \ln \frac{1-\frac{\mathrm{D}}{m-1} \frac{\mathrm{F}\left(\mathrm{T}_{\mathrm{n}}\right)}{\mathrm{T}_{\mathrm{n}-1}}}{1-\frac{m \mathrm{D}}{m-1} \frac{\mathrm{F}\left(\mathrm{T}_{\mathrm{n}}\right)}{\mathrm{T}_{\mathrm{n}-1}}} \quad \text { (eq. (1), Part III, introduced) } \\
& \fallingdotseq \alpha \ln \frac{1-\mathrm{DM} \frac{\mathrm{F}\left(\mathrm{T}_{\mathrm{n}}\right)}{\mathrm{T}_{\mathrm{n}}}}{1-\mathrm{DM} m \frac{\mathrm{F}\left(\mathrm{T}_{\mathrm{n}}\right)}{\mathrm{T}_{\mathrm{n}}}}\left(M=\frac{1}{m-1} ; \mathrm{T}_{\mathrm{n}-1} \fallingdotseq \mathrm{T}_{\mathrm{n}}, \text { assumed }\right) \\
& \Delta \mathrm{t}_{\mathrm{n}}=\alpha \ln \frac{i_{r}+\beta_{\mathrm{r}} \mathrm{T}_{\mathrm{n}-1}-i_{r}}{i_{r}+\beta_{\mathrm{r}} \mathrm{T}_{\mathrm{n}-1}-i_{r} m f\left(\beta_{\mathrm{r}}\right) \mathrm{F}\left(\mathrm{T}_{\mathrm{n}}\right)} \\
& =\alpha \ln \frac{\beta_{\mathrm{r}} \mathrm{T}_{\mathrm{n}-1}}{\beta_{\mathrm{r}} \mathrm{T}_{\mathrm{n}-1}-i_{r} m f\left(\beta_{\mathrm{r}}\right) \mathrm{F}\left(\mathrm{T}_{\mathrm{n}}\right)} \quad\left(i_{r} \text { neglected against } i_{r} m f\left(\beta_{\mathrm{r}}\right) \mathrm{F}\left(\mathrm{T}_{\mathrm{n}}\right)\right)
\end{aligned}
$$

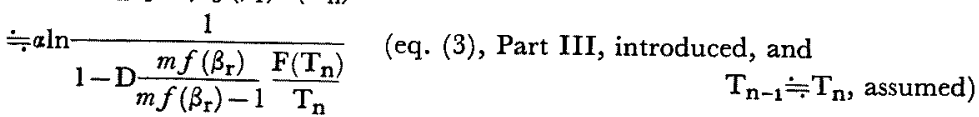

$$
\begin{aligned}
& \fallingdotseq \alpha \ln \frac{1}{1-\mathrm{DN} \frac{\mathrm{F}\left(\mathrm{T}_{\mathrm{n}}\right)}{\mathrm{T}_{\mathrm{n}}}}\left(\mathrm{N}=\frac{m f\left(\beta_{\mathrm{r}}\right)}{m f\left(\beta_{\mathrm{r}}\right)-1}\right)
\end{aligned}
$$

The quantity $\frac{F\left(T_{n}\right)}{T_{n}}$ represents the mean rate of $i_{r}$ or $m$-rise. If this gets larger with $\mathrm{T}$, then $\Delta \mathrm{t}$ doing so too, the repetitive response will cease after a certain while. But if it decreases sufficiently rapidly or if, in addition, a larger $\beta$ is chosen, then the response will continue on without cessation. The practical necessary condition for this is that the rate of rise of $i_{r}$ or $m$ gets smaller with time. The logarithmic nature of $m$-rise suggested above, will probably satisfy this condition, to establish Hoffmann's observation $^{10)}$, provided that $\alpha$-rise is relatively little.

\section{Discussion}

1. $\beta_{\mathrm{r}}$ and $\mathrm{I}_{\infty-\gamma} \gamma$-line, and their probable independence of $\mathrm{I}_{\mathrm{r}}$-rise.

In Part II, it was shown that $\beta_{\mathbf{r}}$ and $\mathrm{I}_{\infty}-\gamma$-line can be the case without threshold rise; in Part III that threshold-rise, if it occurs, disturbs the appearance of $\beta_{\mathrm{r}}$ of a reasonable value, and of the linear relationship between $I_{\infty}$ and $\gamma$. These results, together with the fact that $I_{r}$ is certainly finite at $\beta_{\mathrm{r}}$ in contradiction to the theory of accommodation, point to independence 
of the threshold from $\beta_{\mathrm{r}}$ and $\mathrm{I}_{\infty}-\gamma$-line.

2. $\mathrm{I}_{\mathrm{r}}$-variation and its probable dependence on $m$-rise.

It is sure that $I_{r}$ varies according to conditions. But as far as examined above, it seems to be $m$ that changes in electrotonus, temperature varied, in varied $\mathrm{Na} \cdot$-concentration of the bathing fluid, and also in repetitive responses to constant and linearly rising currents. The only exception encountered is the $\mathrm{I}_{\mathrm{r}}$-rise observed near $\beta_{\mathrm{r}}$, where the rise of $i_{r}$ appeared responsible. One possibility might be that $i_{r}$ changes in short-lasting while $m$ changes in long-lasting exposure to new conditions. There are, however, also reasons to believe that the actual rise of $I_{r}$ near $\beta_{r}$ might have been much smaller than observed: 1) the time of ascent (up to the $\mathrm{I}_{\mathrm{r}}$-level) is only several ten msec in most cases, which seems to be too short to exhibit any appreciable depressive action, 2) in Sugi's experiment ${ }^{11)}$ with linearly rising current, the range of constant threshold was so wide that even $\beta_{\mathbf{r}}$ could hardly be confirmed, and 3 ) in stimulation with exponentially increasing current, the excitation always took place at an almost constant level of potential ${ }^{1,3)}$. All these facts point to threshold constancy in stimulation with slowly rising currents. I dare suppose: was the $I_{r}$-rise near $\beta_{\mathrm{r}}$ not an artifact, at least in part, due to polarization still remaining on isolated fibres? Was the actual rise not much smaller and this small rise not due to rise of $m$ ? This might be a wilful supposition, but if it is correct, and if we can put $f\left(\beta_{\mathbf{r}}\right) \fallingdotseq 1$, then eq. (1) and (3) in Part III are both reduced to eq. (1) or (2) in Part II which provide a reasonable value of $\beta_{\mathrm{r}}$; and eq. (2) and (4) in Part III to eq. (5) in Part II, which expresses a strict linearity of $\frac{I_{\infty}}{I_{r}}-\gamma$-curve. A unific explanation can thus be presented by rise of $m$. Further, the rise of $m$, thus supposed, needs be just small. If it is large, it makes $\beta_{\mathrm{r}}$ too large, $\frac{\mathrm{I}_{\infty}}{\mathrm{I}_{\mathrm{r}}}-\gamma$-curve not linear, the total adaptation time for constant current too short, and the ever-lasting repetitive responses to linearly rising current with $\beta>\beta_{\mathrm{r}}$ impossible. It is quite interesting in this connexion that just a $5 \%$ increase of $m$ was found enough for explaining the facts in C.E.T., as stated above.

\section{3. "Accommodation" in terms of $\mathbf{I}_{\mathbf{r}^{-}}$or $m$-rise}

"Accommodation" is certain as long as a rise of $I_{r}$ is meant by it. In view of the present theory, however, $\mathrm{I}_{\mathrm{r}}$ is different from $i_{r}$, and the rise of $I_{r}$ is highly probably due to rise of $m$. The so-called accommodation becomes then a matter of initiation of impulse having nothing to do with $i_{r}$, the rheobase for excitation. I dare propose therefore to define accommodation practically by rise in $\mathrm{I}_{\mathrm{r}}$, or theoretically by rise in $m$, and to call $m$ the "constant of propagation" for convenience. 
The point is whether the distinction of excitation from initiation of impulse is correct or not. However, the identification of these two has no basis, either. If the distinction has any reason, and the accommodation is really a matter concerning initiation of impulse, then all the explanations for the mechanism of accommodation on the basis of $i_{r}$-rise, including the modern one of Hodgkin ${ }^{12)}$, though to be questioned or reconsidered.

\section{Summary and Conclusions}

1. $\beta_{\mathrm{r}}$ of the observed value and the linear relationship between $\frac{\mathrm{I}_{\infty}}{\mathrm{I}_{\mathrm{r}}}$ and $\gamma$ cannot be expected from the present theory, unless the rise of $I_{r}$ is very small.

2. $\beta_{\mathbf{r}}$ and $\frac{\mathrm{I}_{\infty}}{\mathrm{I}_{\mathbf{r}}}-\boldsymbol{\gamma}$-line are very probably independent of $\mathrm{I}_{\mathbf{r}}$-rise.

3. Variation of $m$ supposed to occur to a small extent, was shown to be very useful for explaining various experimental facts. The rise of $I_{r}$ near $\beta_{\mathrm{r}}$ was supposed to be due partly to artifact, and partly to rise of $m$.

4. It was proposed to define accommodation by rise in $\mathbf{I}_{\mathbf{r}}$ or $m$, and to call $m$ the constant of propagation.

5. Discussions were made on 1) probable independence of $\beta_{\mathrm{r}}$ and $\frac{I_{\infty}}{I_{r}}-\gamma$-line from $I_{r}$-rise, 2) probable dependence of $I_{r}$ on $m$, and 3) accommodation.

6. General conclusions from Part I, II and III.

1) Distinction between excitation and initiation of impulse is very useful for explaining experienced facts.

2) The rise of $m$, not of $i_{r}$, to a small extent seems to be a general basic event that happens in normal nerves in various conditions.

3) Of the well-known three factors of stimulating currents, a) intensity $\left(>i_{r}\right)$, b) duration ( $>$ utilization time) and c) slope $\left(>\beta_{\mathbf{r}}\right)$, the third one appears to be an additional requisite for initiation of impulse.

I express my sincere thanks to Dr. K. Konishi and Mr. S. Tsukui for their kindest help in preparing the manuscripts.

\section{References}

1) Diecke, F., Z. Naturf., 1954, 9b, 713.

2) Tasaki, I., Jap. J. Physiol., 1950, $1,1$.

3) Frankenhäuser, B., Cold Spring Harbor Symp. Quant. Biol., 1952, XVII, 27.

4) Suzuki, M., Seirigaku Koza (Jap.), II, 1, B, Nakayama, Tokyo, 1952.

5) Yamagiwa, K., Jap. J. Physiol., 1956, 6, 167.

6) Tasaki, I., ibid., 1950, 1, 7.

7) Tasaki, I., Cytologia, 1950, 15, 219. 
8) Granit, R. \& Skoglund, C. R., J. Neurophysiol., 1943, 6, 337.

9) Katz, B., J. Physiol., 1936, 88, 239.

10) Hoffmann, P., Arch. Anat. Physiol., Lpz., 1910, 1910, 247.

11) Sugi, Y., Nihon Seirigaku Z. (Jap.), 1939, 4, 95.

13) Hodgkin, A. L. Biol. Rev. 1951, 26, 339. 